\title{
烈 RAIN SENSING AUTOMATIC CAR WIPER USING 555 TIMER
}

\author{
Moni Sree $\mathbf{H}^{1}$, Dharshana $\mathbf{N}^{2}$, Dheetchithaa $\mathrm{S}^{3}$, Archana $\mathrm{A}^{4}$ \\ E-Mail Id: monisree.18ec@kct.ac.in \\ Department of Electronics and Communication Engineering, Kumaraguru College of Technology, \\ Coimbatore, Tamil Nadu, India
}

\begin{abstract}
Over the past few years the automotive industry has aggressively researched ways to develop systems for safety, to save humans life. Generally working of a car wiper is based on manual switching. In this case the driver has to switch ON the power button to turn on the wiper. It complicate things because the driver has to concentrate simultaneously on driving and wiper. With drivers exposed to increased distraction, automatic rain sensing wiper system becomes more appealing feature, as they eliminate the risk and distraction to turn on the wiper manually. In this automatic wiper system, a rain sensor, microcontroller and a driver integrated circuit is used to convert manual operation into automatic operation.

When water falls on the rain sensor, the sensor sends the signal to the microcontroller and then the microcontroller processes the data and energizes the driver IC to turn on the wiper. With these modifications automatic cleaning of the glass can be done without the involvement of the driver.
\end{abstract}

Keywords: Automotive, Drivers, Eliminate risk, Manual operation, Wiper.

\section{INTRODUCTION}

This article guides a stepwise walkthrough by Experts for writing a successful journal or a research paper starting from inception of ideas till their publications. A car wiper is a device which is used to remove droplets of rainwater from a windshield of a car.

A wiper generally consists of a metal arm and a long rubber blade to wipe the screen. In some vehicles, pneumatic power is used. Here, the metal arm gets powered by battery. The blade moves in clock-wise and counter clock-wise direction on the glass, pushing the water from the surface of the screen. Modification of speed is automatically done based on the amount of the rainfall. Two synchronized radial type arms are used in most of the automobiles, whereas panto-graph(parallelogram) arms are used in commercial automobiles. Wipers are

automated in many ways. In this, we propose an unmanned wiper which senses rain and starts automatically and switches off automatically when the rain stops besides manual off is also provided. By using this, there will be no need for physical intervention of human to control the speed of the wiper.

For this purpose, we use a rain sensor to detect the rain and then the signal is managed by motor driver and takes the required action.

Over the last ten years, the advancement in the automobile industry has been increased to find modern techniques to increase safety. There are many reasons behind the vehicles which are not equipped with automatic car wipers. Many reasons in the sense, the car wipers are too expensive to fit in economical automobiles and they are too unreliable for new automobiles. Many automobile companies made an attempt to construct the automatic car wiper at low cost which is not only economical but also efficient they actually failed. This paper is all about the attempt they tried to construct and bring out the correct methodology for the automatic wiper.

\section{NEED FOR AUTOMATIC WIPER SYSTEM}

In the present automobiles the number of facilities is much higher. The driver has to concentrate on road while driving, and with increased traffic, things get complicated. The features in the car is increased for the purpose of man like GPRS to trace the route, music system, air condition system etc. may drive away the attention of the driver in many ways.

Thus, an effort has been made to reduce the effort of driver. Since this system is put into use in many higher end cars and has been successfully working, an effort was made to reduce the cost of the system so that this system can be implemented in common economic cars where a common man can also enjoy the benefits.

\section{OBJECTIVE}

The following are the objectives of the project: -

$>$ To reduce the risk of accidents

$>$ To automate the wiper mechanism and working in automobiles.

$>$ To reduce the driver's tasks and allow driver to concentrate on driving.

$>$ To reduce the operations of the wiper, in manual mode

DOI Number: https://doi.org/10.30780/IJTRS.V05.I08.003 


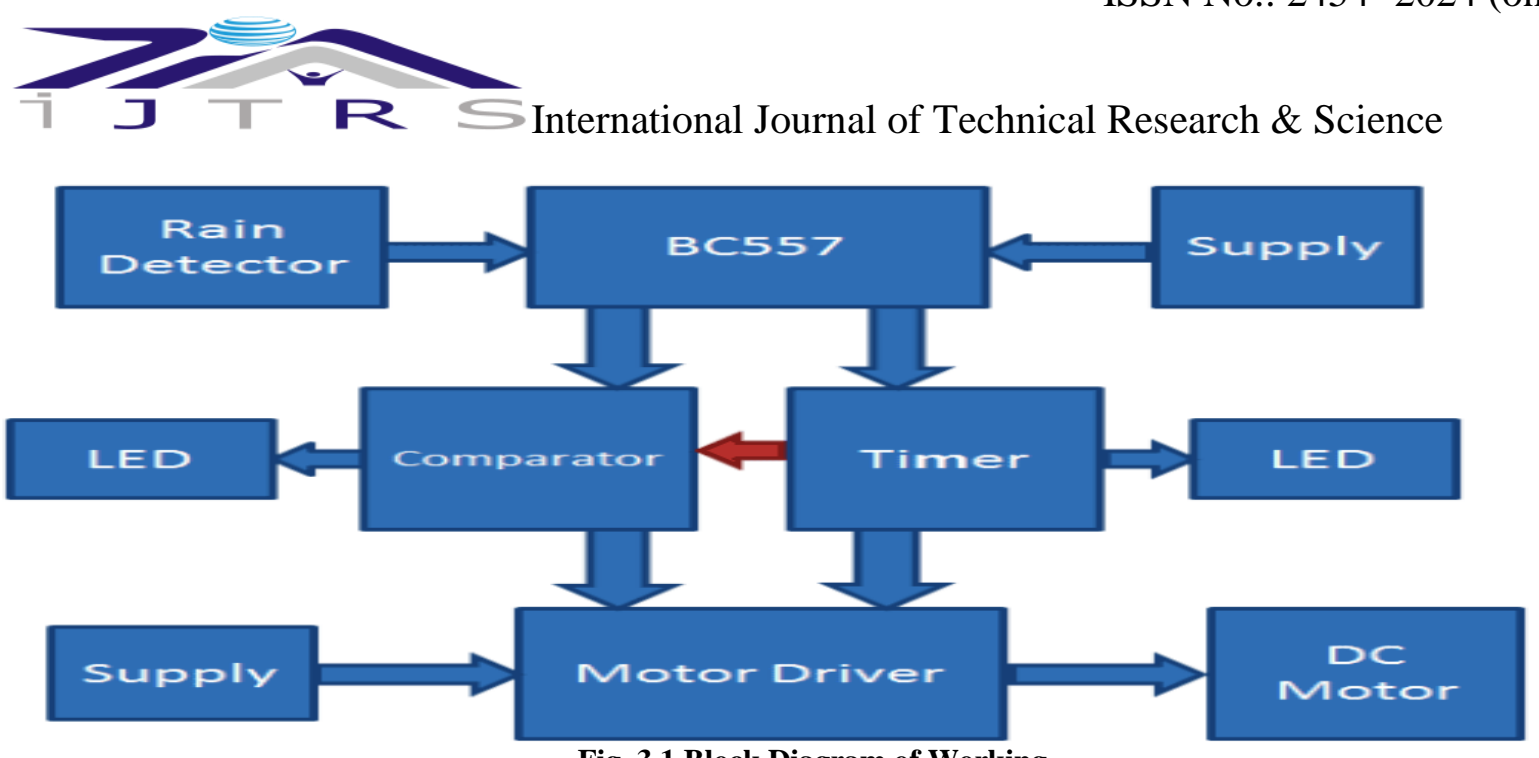

Fig. 3.1 Block Diagram of Working

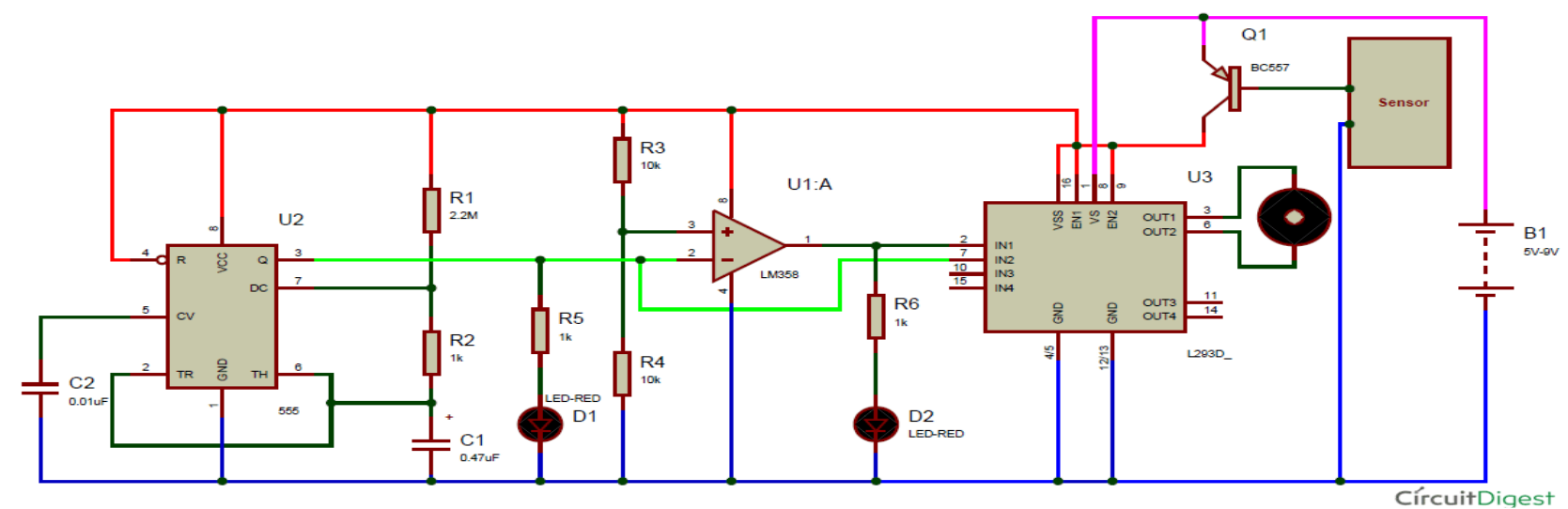

\subsection{Stages Involved}

Fig. 3.2 Circuit Diagram

This Automatic Rain Sensing Wiper Control Circuit can be divided into four parts. First part includes 555 IC in Astable Mode, second part includes Comparator, third part has Motor Driver circuitry and forth part is Rain Detector.

\section{LITRATURE SURVEY}

In this work, they proposed an automated wiper manage machine that's economical, efficient and has an excellent output. This paper uses a resistive rain sensor. They developed a wiper which is practically demonstrated and a sensor is advanced that's an equivalent mathematical version. The rain sensor normally has a predetermined rotational geometry, when the droplets of the rainfall on the sensor, the droplets forms a layer at the floor of the sensor inflicting non linearity to its resistance. To lower the non-linearity and to growth the efficiency of the gadget, the reaction from the sensor is to be linearized. The response can be linearized by using the linearized circuit with the equivalent electrical version of the sensor. To reap the changes inside the speed based in the output provided by using the rain sensor, customized PIC micro-controller is used inside the paper named "“A novel and cost-effective resistive rain sensor for automatic wiper manage: circuit modeling and implementation” through Mukul Joshi, M. A Joshi, Vinayak Sagare, D. N. Sonawane.

In this paper, fuzzy common sense become used to operated the collected analog information from the rain sensor. The program turned into programmed to apply fuzzy logic in collecting records. The wiper motor is controlled by the microcontroller which uses pulse width modulation (PWM). The reason for the usage of the bushy common sense in this is only because it is able to be without problems rearrangeable. They are used to decorate things. It can be used to construct specific wiper for exclusive cars without changing any hardware configuration. This is primarily based at the paper named "Design and implementation of a reconfigurable computerized rain sensitive windshield wiper" via Lubna Alazzawi, Avik Chakravarty.

In this paper, the Bluetooth vehicle wiper gadget is proposed. The wiper begins to wipe when it captures any wifi Bluetooth signal from any cellular. They brought an innovative way to wipe the windshield. Although it's far a DOI Number: https://doi.org/10.30780/IJTRS.V05.I08.003

pg. 22 www.ijtrs.com www.ijtrs.org 


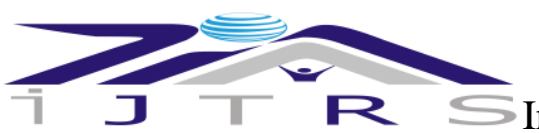

\section{International Journal of Technical Research \& Science}

Bluetooth based car wiper, there may be a need for human intervention. For the wiper to begin, it wishes to detect Bluetooth signals and people must be sent with the aid of the driver with a purpose to start the wiper. The Bluetooth car wiper is constructed the usage of HC05 Bluetooth, Arduino and servo motor. We can control the movement of the automobile wiper via sending low range Bluetooth signals. This painting is done inside the paper named "Arduino based Bluetooth operated vehicle wiping method using android cellular phone" by using Sourish Mitra, Soham Biswas, Mrinmoy Aus.

\section{EXISTING SYSTEMS}

Every year during the rainy season, greater than two million humans die worldwide due to accidents within the wet season in accordance to world health organization. People grow to be dying because of small mistakes. Today's automobile wipers need human intervention to begin the wiper and to control its speed. In this type of manual switching, the driving force needs to exchange on the wiper when wanted and need to alter the speed of the wiper as required. This causes inconvenience to the motive force all through rainfall.

\section{METHODOLOGY}

For Astable Multivibrator, we've got used a 555 Timer IC for generating pulse in each 2-three seconds (depends on capacitor value), way 555 Timer IC is configured in Astable mode. Output of Astable Multivibrator is immediately connected to inverting pin of Comparator LM358 and Pin No 7 of Motor Driver L293D. Output of comparator is at once related at pin 2 of motor motive force IC. Comparator LM358 IC is used right here for evaluating 555 timer IC's output voltage and reference voltage across comparator's non inverting terminal, set by using Voltage Divider Circuit . Two LEDs were used, one on the output of 555 Astable circuit and other on the output of comparator. A Water Detector or Rain Sensor is used for detecting the water or rain. Output of Astable Multivibrator and Comparator is applied to motor driver, in order to similarly power the wiper motor. Whole circuit may be powered the use of $5 \mathrm{v}-12 \mathrm{v}$ battery depending upon the application.

\section{DETAILS OF THE COMPONENT}

\subsection{Timer IC}

The 555 timer IC was first presented around 1971 by the Signetics Corporation as the SE555/NE555 and was designated "The IC Time Machine" and was additionally the absolute solitary business clock IC accessible. It given circuit originators a generally modest, stable, and easy to understand incorporated circuit for clock what's more, multivibrator applications. It works from a wide scope of intensity supplies running from +5 Volts to +18 Volts supply voltage. Sinking or sourcing $200 \mathrm{~mA}$ of burden current. The yield of a 555 timer can drive a transistor-transistor rationale (TTL) because of its high current yield. The obligation pattern of the timer is movable. The 555 timer joins an unwinding oscillator, two comparators, an R-S flip-flop, and a release capacitor. In any case, if the edge input (pin 6 ) is presently raised above $+(2 / 3) \mathrm{VCC}$, the yield will come back to ground and the release transistor will be turned on once more. At the point when the edge input comes back to ground, the IC will stay right now, was the first state at the point when we began this investigation. The least demanding approach to permit the limit voltage (pin 6) to progressively ascend to $+(2 / 3)$ VCC is to interface it remotely to a capacitor being permitted to charge through a resistor $\mathrm{R}$ and $\mathrm{C}$ values can be adjusted in this way for almost any time interval we get.
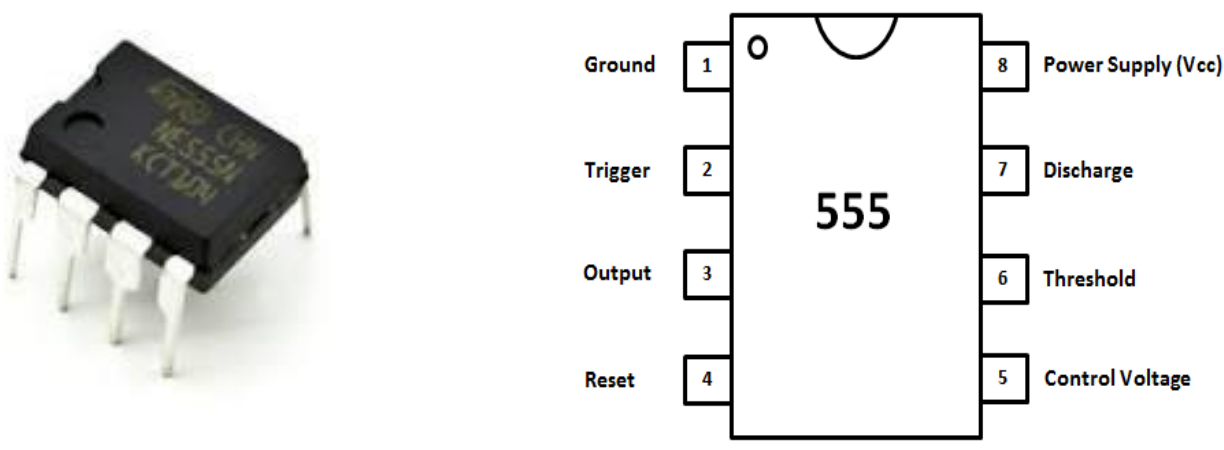

Fig. 7.1 555 IC

\subsection{L298D IC - Motor Driver}

The L298 is an integrated monolithic circuit in a 15-lead Milliwatt and PowerSO20 packages. It is a high voltage, high current dual full-bridge driver designed to accept standard TTL logic levels and drive inductive loads such as 


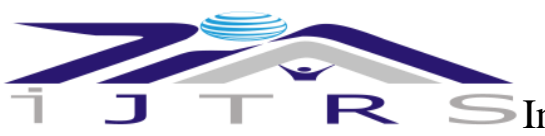

International Journal of Technical Research \& Science

relays, solenoids, DC and stepping motors. Two enable inputs are provided to enable or disable the device independently of the input signals. The emitters of the lower transistors of each bridge are connected together and the corresponding external terminal can be used for the connection of an external sensing resistor. An additional supply input is provided so that the logic works at a lower voltage. L-298 has two enable input to control any device by enabling or disabling it. L 298 IC is most commonly used to make motor drivers or motor controllers. These motor controllers can be controlled by any micro controller e.g. Arduino, PIC, Raspberry Pi etc. They receive input from micro controllers and operate the load attached to their output terminals correspondingly. L-298 motor driver (HBridge) is able to control two different DC motors simultaneously. While it can control a single stepper motor as well. L 298 has two Pulse Width Modulation (PWM) pins. PWM pins are used to control the speed of the motor. By changing the voltage signal's polarity at its input. we can rotate the motor in either clockwise or counter clockwise direction.

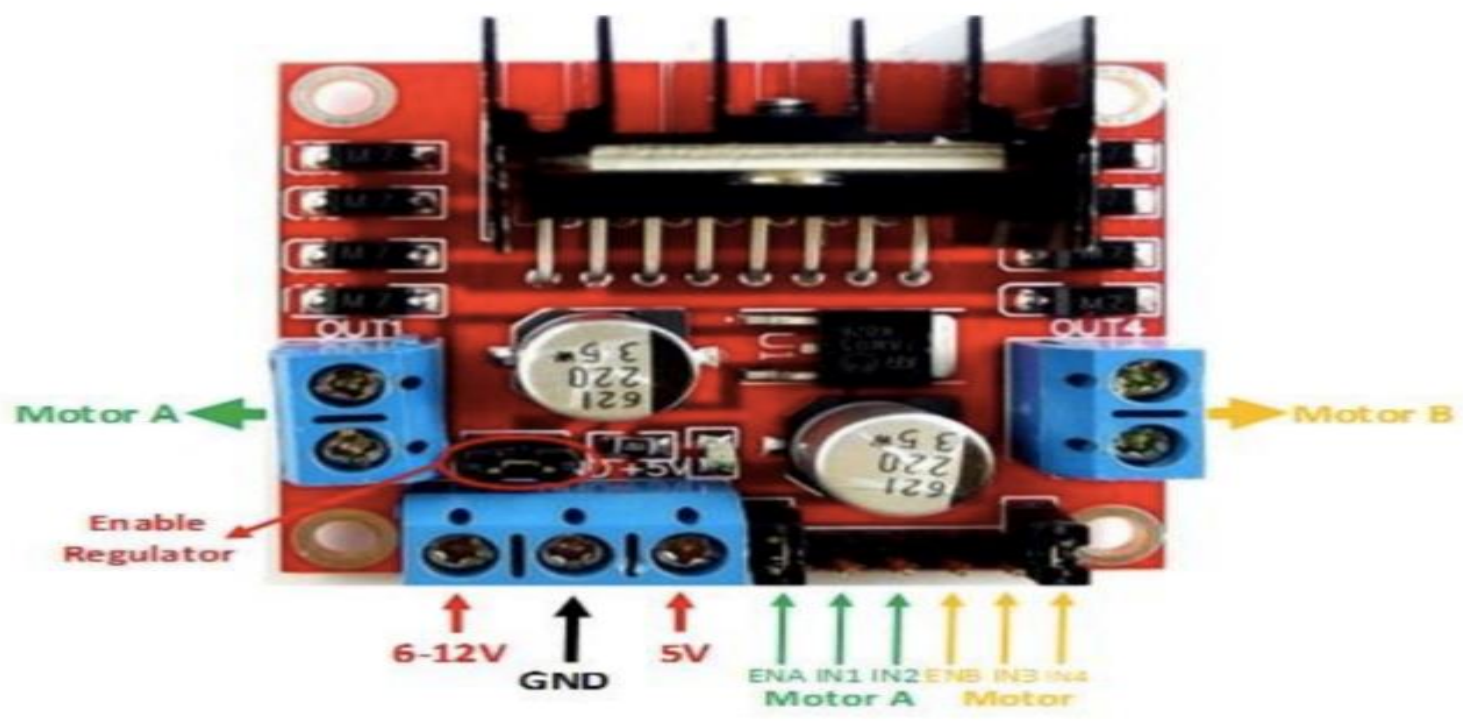

\subsection{LM358}

Fig. 7.2 Motor Driver

The LM358 is a duel operation amp, which means there's two operation amps in a single IC. One of the less difficult circuits and normally utilized circuits are the operation amp comparators. A comparator fundamentally thinks about two voltages and if one is more prominent than the other the yield of the operation amp is either ON or OFF. Comparators have discovered numerous utilizes in Day Night switches, basic Analog to Digital Converters, electronic indoor regulators and even directed force supplies. There are two segments we should work with so as to decide at what voltage will it switch ON furthermore, at what voltage it will turn OFF. Detecting voltage will be the information voltage, this voltage could fall off an operational enhancer yield if the genuine sensors changes are too little to even consider working with. On the off chance that we have the detecting voltage made through a potentiometer we may disregard utilizing an operation amp to enhance any flag as we will have a sensible voltage distinction to work with. The detecting voltage can be applied to the non-modifying input, this will guarantee that once the detecting voltage has surpassed the reserch Reference voltage that must be set on the modifying input the operation amp will turn ON. In the event that we apply the detecting voltage to the reversing input also, the reference voltage to the non-reversing input we will have the operation amp OFF when the detecting voltage is over the reference voltage and the operation amp ON when the detecting voltage is underneath the reference voltage.

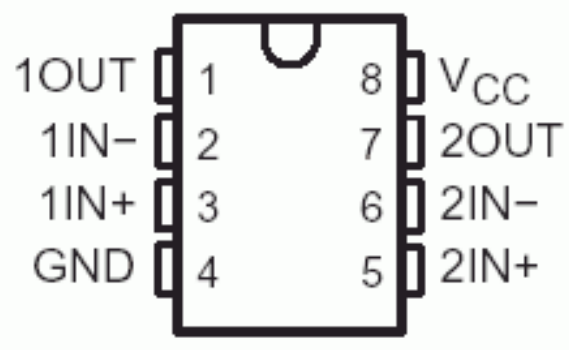

Fig. 7.3 LM358

DOI Number: https://doi.org/10.30780/IJTRS.V05.I08.003

pg. 24

Www.ijtrs.com

www.ijtrs.org

Paper Id: IJTRS-V5-I6-002 


\subsection{Rain Sensor}

Rain sensor is one sort of exchanging gadget which is used to identify the precipitation. It works like a switch and the working guideline of this sensor is, at whatever point there is rain, the switch will be ordinarily shut. The rainsensor module board incorporates nickel covered lines and it deals with the obstruction standard. This sensor module licenses to measure dampness through simple yield pins and it gives an advanced yield while dampness limit outperforms.

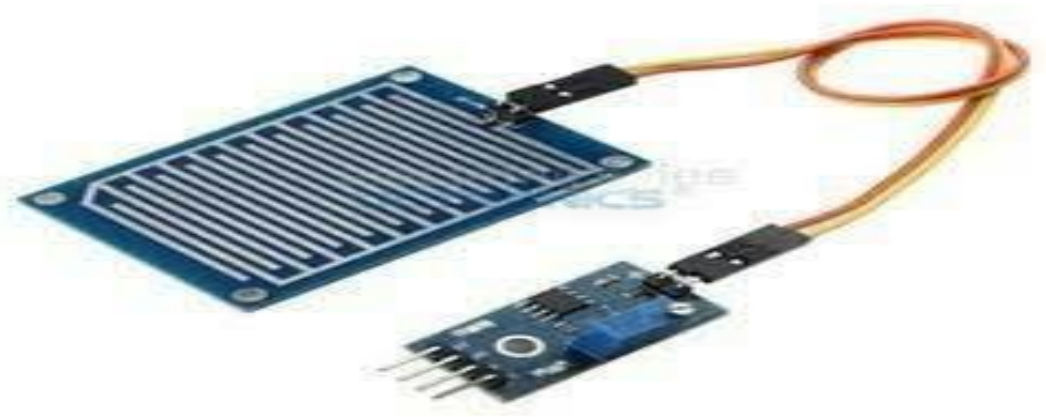

\section{IMPLEMENTATION}

Fig. 7.4 Rain Sensor

Working of this Automatic Rain Sensing Car Wiper challenge is simple. As we already defined that this circuit has four elements namely Astable Multivibrator, Comparator, Motor Driver and Rain Detector. When water drops of rain falls over the Rain Sensor then it'll trigger the PNP transistor BC557 and PNP transistor turns ON the electricity deliver of entire circuit and circuit begin working until there's water at the Rain Sensor. Now after the energy deliver has been became ON, Astable Multivibrator starts oscillating in configured frequency. Now when the output of 555 Timer IC is going HIGH then the comparator LM358 offers LOW output and when the output of 555 IC goes LOW then the Comparator's output is going HIGH. And by the usage of these two outputs DC motor turns clock smart and anticlockwise and wiper connected to it turns proper to left and left to proper, through Motor Driver IC L293D. That is how the wipers automatically feel rain and gets activated. They continue to be activated till there's water on Rain sensor, as soon as the water evaporates wipers get stopped. Two LEDs are also used here used for indication.

\section{RESULTS}

To make the wiper on automatically is easy but interfacing it with the application is matters.

The expected output is to make the wiper on by sensing the rain water effectively.

These points will act as future scope.

$>\quad$ This system also useful in-home applications like cleaning the window glasses and it intimates the rainfall and also notify people in the house. So that people can take care of things like clothes, food grains and products.

$>\quad$ The usage of better speed control mechanisms will guide wiper more effectively and reduce the consumption of battery power.

\section{CONCLUSION}

This low-cost automation is dependable and may be included in Economic magnificence cars. Automation with variable frequency may be included with incumbent wiper system.

The following disadvantages are inferred in the course of the project simulation.

$>$ The Nickel coating is getting oxidized and rust is forming on the surface which may additionally impede the sensing aptness of the Rain sensor module.

$>\quad$ The sensor gives best 3 set of analogue values for the numerous rain intensities. Thus, with this sensor best 2 variables speed are feasible.

Thus, this low price and integrated computerized wiper machine with variable frequency greatly reduces the avert for the driver. By the use of this device, the user can also manage manually along with automated control of the wiper system. The Older model of automobile wiper gadget has been upgraded. Users do not need to face problems in controlling the wiper when its miles raining heavily. When the engine is started, the sensor circuit is functioned and it is controlled via a microcontroller. 


\section{REFERENCES}

[1] Automatic Rain Operated Wiper System in Automobile: A Review by Kothari Mohit, Shah Amit, Patel Vipul and Kadakia Nishant in International Journal for Scientific Research \& Development| Vol. 3, Issue 02, 2015| ISSN:2321-0613.

[2] Automatic control of vehicles: An Analysis by Kushal Sarin, Jatin Sethi, Anshuman Gupta, Usha Tiwari and Ishan Mathur in International Journal of Advanced Research in Computer and Communication Engineering April 2013.

[3] Review report on soc on various platforms for vehicles by Sheeja S. Suresh and Anuradha S. Joshi1 in International Research Journal of Engineering and Technology.

[4] Rainy Weather Recognition from in-vehicle Camera Images for Driver Assistance by I. Ide, Y. Mekada, H. Murase, Y. Tamatsu, H. Kurihara, T. Takahashi, and T. Miyahara, in IEEE Intelligent Vehicles Symposium, 2005.

[5] KadakiaNishant, A Kothari, Mohit A Shah, Amit V Patel Vipul R: Automatic Rain Operated Wiper System in Automobile, International Journal for Scientific Research \& Development Vol. 3, Issue 02, 2015.

[6] AHM FazleElah and Mohammad ShafiurRehman in Intelligent Windshield for Automotive Vehicles 17th International Conference on Computer and Info. Technology 22-23 December 2014. International University, Dhaka Bangladesh.

[7] H. Kurihara, T. Takahashi, I. Ide, Y. Mekada, H. Murase, Y. Tamatsu, and T. Miyahara, "Rainy Weather Recognition from in vehicle Camera Images for Driver Assistance," In IEEE Intelligent Vehicles Symposium, 2005, pp. 205-210.

[8] Anuradha S. Joshi1, Sheeja S. Suresh, "review report on soc on various platforms for vehicles", International Research Journal of Engineering and Technology (IRJET).

[9] Tapan S. Kulkarni, Harsh S. Holalad, July 2012, "Semi-Automatic Rain Wiper System," International Journal of Emerging Technology and Advanced Engineering, ISSN 2250-2459, Volume 2, Issue 7.

[10] N. M. Z. Hashim, July 2013. "Smart Wiper Control System," International Journal of Application or Innovation in Engineering \& Management (IJAIEM), ISSN 2319 - 4847, Volume 2, Issue 7.

[11] K. V. Viswanadh, January-2015, "Design \& Fabrication of Rain Operated Wiper Mechanism using Conductive Sensor Circuit," International Journal of Engineering Research \& Technology (IJERT), ISSN: 2278-0181, Vol. 4 , Issue 01. 